\title{
HOW TO DETERMINE THE EIGENVALUES OF G-CIRCULANT MATRICES
}

\author{
ERIC NGONDIEP
}

Abstract. For a given nonnegative integer $g$, a matrix $C_{n, g}$ of size $n$ is called $g$-circulant if $C_{n, g}=\left[a_{(r-g s) \bmod n}\right]_{r, s=0}^{n-1}$. Such matrices arise in wavelet analysis, subdivision algorithms, and more generally when dealing with multigrid/multilevel methods for structured matrices and approximations of boundary value problems. In this paper, we study the eigenvalues of $g$ circulants. The relationship to the harmonic analysis is explored and based on the new recursive formulas for eigenvalues of such class of matrices are obtained. This result represents an extension of the work due to E. Ngondiep and S. Serra Capizzano in establishing bounds for preconditioners for the linear system of equations determined by the same matrix and it could be seen as a tool for the analysis of the preconditioners. Numerical experiments are presented to illustrate the theoretical result. Mathematics subject classification (2010): 65F10, 15A18.

Keywords and phrases: Circulants, $g$-circulants, Euler indicator, recursive procedure, eigenvalues.

\section{REFERENCES}

[1] C. M. Ablow And J. L. BRENner, Roots and canonical forms for circulants matrices, Trans. Amer. Math. Scot. 107: 366-376 (1963).

[2] A. Aricò, M. Donatelli, S. Serra-Capizzano, V-cycle optimal convergence for certain (multilevel) structured linear systems, SIAM J. Matrix Anal. Appl. 26-1 (2004), pp. 186-214.

[3] O. Axelsson, V. A. BARKER, Finite element solution of boundary value problems: theory and computation, Society for Industrial and Applied Mathematics (2001).

[4] J. L. BRENNER, g-circulant matrices over a field of prime characteristics, Illinois, J. Maths, Vol. 7, Issue 1 (1963), 1-179.

[5] J. L. BRENNER, Mahler matrices and the equation QAAQ, Duke Math. J. Vol. 29 (1962), pp. 13-28.

[6] R. H. CHAN, M. K. NG, Conjugate gradient methods for Toeplitz systems, SIAM Rev. 38 (1996), pp. 427-771.

[7] P. Davis, Circulant Matrices, J. Wiley and Sons, New York, (1979).

[8] M. Donatelli, S. Serra Capizzano, D. Sesana, Multigrid methods for Toeplitz linear systems with different size reduction, BIT (2011) 1-23.

[9] C. Estatico, E. Ngondiep, S. Serra Capizzano, D. Sesana, A note on the (Regularizing) preconditioning of $g$-Toeplitz sequences via $g$-circulants, JCAM 236 (2012), pp. 2090-2111.

[10] D. FASInO, P. TILLI, Spectral clustering properties of block multilevel Hankel matrices, Linear Algebra Appl. 306 (2000), pp. 155-163.

[11] B. Friedman, Eigenvalues of composite matrices, Proc. Cambridge Philo. Sot. 57: 37-49 (1974).

[12] W. HackBush, Multigrid methods and applications, Springer-Verlag, New York, (1979).

[13] E. NGONDIEP, Distribution in the sense of eigenvalues of $g$-Toeplitz sequences: clustering and attractions, Arab J. Math. Sci. 22 (2016), 45-60.

[14] E. Ngondiep and S. Serra Capizzano, Approximation and spectral analysis for large structured linear systems, LAP LAMBERT Academic Publishing, ISBN-13: 978-3-8454-1547-5, ISBN10: 3845415479, EAN: 9783845415475 , October 2011, 268 pages.

[15] E. Ngondiep, S. Serra CapizZano, D. Sesana, Spectral features and asymptotic properties for $\alpha$-circulants and $\alpha$-Toeplitz sequences: theoretical results and examples, preprint available from http://arxiv.org/abs/0906.2104 (2009). 
[16] E. NGOndiep, S. SERra CAPIZZAno, D. SESANA, Spectral features and asymptotic properties for g-circulants and g-Toeplitz sequences, SIAM J. Matrix Anal. Appl. 31-4 (2010), pp. 1663-1687.

[17] S. SERRA CAPIZZANO, The spectral approximation of multiplication operators via asymptotic (structured) linear algebra, Linear Algebra Appl. 424 (2007), pp. 154-176.

[18] S. Serra Capizzano, D. Sesana, A note on the eigenvalues of g-circulants (and of g-Toeplitz, g-Hankel matrices), Calcolo (2014) 51: 639-659.

[19] J. Stoer, R. BULIRSCH, Introduction to numerical analysis: Text in applied mathematics, Second edition, Springer-Verlag, (1991).

[20] G. STRANG, Wavelets and dilation equations: a brief introduction, SIAM Rev. 31 (4), (1991), pp. 614-627.

[21] William F. Trench, Properties of unilevel block circulants, Linear Algebra Appl. 430 (2009), pp. 2012-2015.

[22] W. TRENCH, Properties of multilevel block $\alpha$-circulants, Linear Algebra Appl. 431 (2009), pp. 1833 1847.

[23] U. Trottenberg, C. W. Oosterlee, A. Schüller, Multigrid, Academic press, London (2001).

[24] Cline Plemons Worm, Generalized inverses of certains Toeplitz matrices, LAA 8, 25-33 (1974).

[25] P. ZELLINI, On the optimal computation of a set of symmetric and persymmetric bilinear forms, LAA 2, 3101-119 (1979). 